\title{
Research on the Manufacture of Bamboo Lamp Based on Morphologic Concept and Elements
}

\author{
Yujia Liang \\ Department of Industrial Design \\ Wuhan University of Science and Technology \\ Wuhan, China
}

\begin{abstract}
This paper makes study on the production process and method of bamboo lamps based on different morphological concepts and elements. According to the morphological concept of dot, line, facet and stereo, the paper analyzes the specific morphological characteristics of existing bamboo lamps and lanterns, and studies the production process and methods of existing bamboo lamps. The study on the production process and method of existing bamboo lamps provides realistic basis for the production process and methods of bamboo lamps design and provides theoretical basis for the morphological innovation of bamboo lamps, to promote the development of bamboo lamp design of various forms and processes.
\end{abstract}

Keywords-bamboo lamps; production process; form

\section{INTRODUCTION}

Under the social background of pursuit of green and lowcarbon, bamboo has been widely explored in the world because of its natural, environmental and mechanical properties. The product design, which is based on bamboo material and bamboo technology, can show the unique aesthetic feeling of bamboo, and also promote economic growth and friendly environmental development. The use of bamboo has a long history in China and the bamboo species are abundant, so the bamboo lantern not only has the function of lighting, an more becomes one of the symbols of Chinese culture, which containing the wisdom of oriental nation and their longing for a better life. Bamboo lamps and lanterns is a new application form in the new era.

The analysis on "pattern" of bamboo lamps and lanterns is a separate discussion of its "shape" and "state". In the analysis of the design of bamboo lamps, "shape" refers to the visual boundary and constituent elements, and "state" is the spiritual support of the bamboo lamps as the objective objects. Bamboo is endowed with excellent mechanical properties and can be transformed into dots, lines, facet, stereo and other constituent elements through a variety of processes, to express the designer's spiritual sustenance. Based on the constituent elements of bamboo lamps and lanterns, such as dot, line, facet, stereo, it discusses the transformation method and process of constituent elements in changing bamboo into lamps and lanterns, which provides a beneficial reference for the innovation of bamboo lamps and lanterns.

\section{The Production Process FOR DOT ELEMENT}

"Dot" is the basis of all forms. Dot in the geometry has no specific size, only a specific location; but in the design structure, its location, area, shape can be determined. The dot is visible only by its from, and is a the visual unit with spatial position [1]. "Dot" is the most basic element of all forms and it is extremely important in the morphological structure of bamboo lamps. According to different bamboo production processes, it can be transformed into various forms [2].

\section{A. The Direct Application of Natural Bamboo}

Due to the hollow character of the bamboo in its growth, the raw bamboo can be cut off transversely to form a hollow bamboo tube with a circular cross section. The installation and arrangement of light source can be easily realized in the bamboo tube. In addition, if changing the cutting angle, the bamboo cut off can have an oval cross-section, which enriches the shape of bamboo lamps. However, it should be noted that the raw bamboo will have a solid cross-section at the bamboo joint. Therefore, when selecting raw bamboo for cutting and making lamps, we should try to choose a position far away from the bamboo joint and avoid the position of the solid core, to achieve efficient production and processing.

In the processing of raw materials, according to the different form design of bamboo lamps, we can choose turning and polishing, drilling or direct use, and other use types. For turning and polishing of natural bamboo, the rough outer wall and inner wall of raw bamboo can be polished smooth, the outer skin of bamboo removed, making the outer layer of bamboo show the color and texture of inner wall, so that the design of bamboo lamp has a unity of appearance and texture in visual perception and touch feeling as in "Fig. 1". Drilling is to make a hollow processing of bamboo after cutting it off, so that light shines through the holes hollowed out to avoid direct illumination bringing discomfort to the user, and the arrangement of holes of different sizes can make the bamboo lamps display their rhythmic beauty as in "Fig. 2". The direct use of natural bamboo is to place the light source directly in the bamboo tube, with simpler process and fewer decorative elements, to make them look more pure and original in visual feeling as in "Fig. 3". 


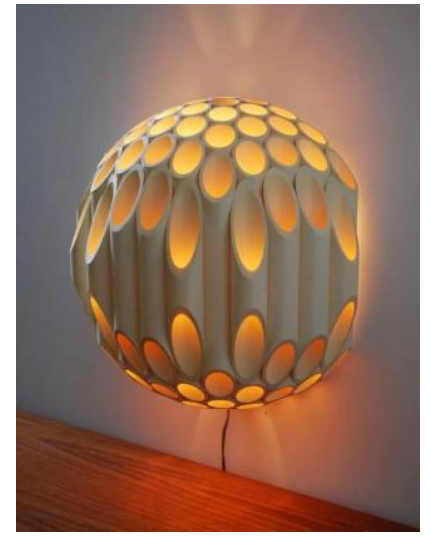

Fig. 1. The bamboo lamp by turning process.

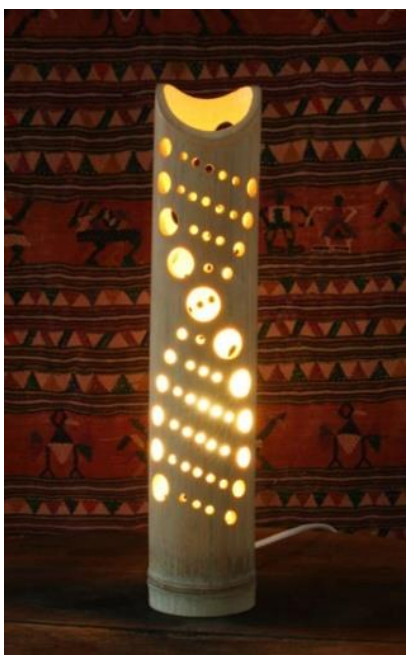

Fig. 2. The bamboo lamp by hollow processing.

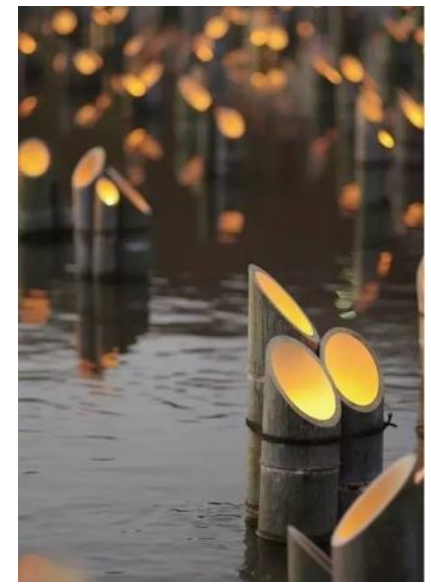

Fig. 3. The arrangement of bamboo lamps and lanterns.

Taking the completed bamboo tube as the basic element, connect some luminous bamboo tubes together by gluing, bundling and other combining type, to bring the designer's ideas into reality, meeting the lighting requirements of lamp design and making bamboo lamp design have more practical value.

\section{B. Processing and Application of Artificial Bamboo}

Artificial bamboo refers to the application of new bamboo produced by hand-made or mechanized processing. With the progress of mechanized production, the raw bamboo can be made into linear bamboo filament, block board and flake bamboo skin by combing, striping, pressing and gluing, and planing.

Bamboo plate drilling and hollowing process, enriches the form of bamboo lamps based on the element of dot, and with the development of technology, the plasticity of bamboo will be continuously improved. In order to achieve different design patterns, the production methods of bamboo lamps by use of artificial bamboo are different. With Shi Daoyu's Bamboo Moon Lamp in "Fig. 4" as an example, for the production process of the pattern, first make turning of the bamboo block and then make rotary cutting to complete the shape of main body, and then drill and hollow the main body to let the light source come out. In the production process of the bamboo plate splicing for chandelier as in "Fig. 5", turning cannot be made for the square object, so first drill and hollow the bamboo plates and then splice them to complete bamboo lamp design.

The production process of artificial bamboo lamp is closely connected with the achievement of product design form. Based on pursuit of the design effect, the convenience of production process plays an important role in the production and use of the product [3]. The appropriate technology can achieve the precise expression of the shape, fully reflect the beauty of the product composition, and present the designer's aesthetic taste.

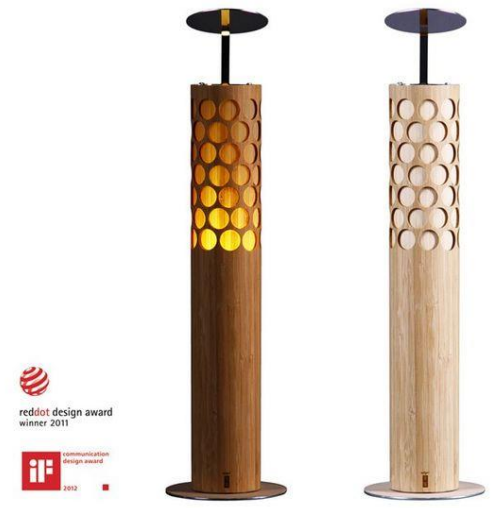

Fig. 4. Bamboo Moon Lamp. 


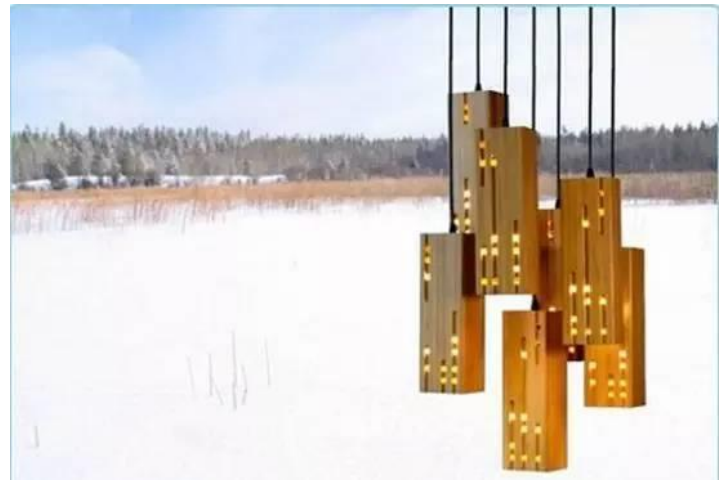

Fig. 5. Hollow chandelier spliced by bamboo plates.

\section{THE PRODUCTION PROCESS OF LINE ELEMENT}

"Line" comes from the movement of "dot". In the definition of geometry, the line refers to the reaction of dot movement, with the position and length, but without the width and thickness, and it is the junction of all the edges of surface and between the surfaces [4]. However, in the product shape, the line needs to be perceived, therefore, the line has a certain width and thickness, but the ratio of width and length is close to infinitesimal. In the design of bamboo lamps, "line" is often presented by the bamboo filament of wiredrawing. Based on the excellent mechanical properties and chemical composition of bamboo, bamboo can be bent into a complex curve to meet the design requirements.

\section{A. Straight Line}

In the design of the product shape, the visual features conveyed by the straight line have unparalleled directionality, hardness, quietness and seriousness. Straight lines has diverse forms, rich expressiveness; the horizontal line gives a sense of calmness and security; plumb line gives brings a towering and hard experience in visual sense, oblique line shapes a downrange and impetuous image in visual sense [5].

In the writings of ancient Chinese literary, bamboo is a symbol of a gentleman, with the cultural connotation of integrity. In the design of bamboo lamp with straight line as element, the straight line not only becomes the main elements of the form, but also makes a close combination of the design with the image of a gentleman in Chinese culture. Therefore, in the design of bamboo lamp with straight line as element, the shapes mostly have a strong Chinese flavor as in "Fig. 6" to achieve the integration of "shape" and "state", and by the visual symbols generated by "shape", people are encouraged to feel the spiritual connotation of design.

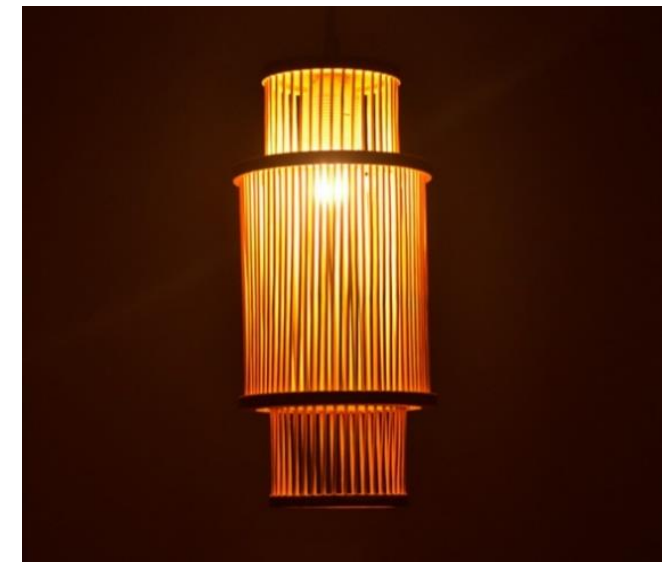

Fig. 6. The bamboo lamp based on straight line.

In the production process, the design of bamboo lamp with straight line as elements is mainly to cut the appropriate bamboo into sections or split into pieces, make wiredrawing and hand-reeling and finally make anti-corrosion treatment and drying, to produce the bamboo filament in accordance with the design. Compared with hand-reeling, mechanical drawing has high accuracy and large output, can ensure the consistency of bamboo wire diameters to the maximum extent, but it loses the fun of bamboo product by hand-reeling. Select the appropriate bamboo wires, and make simple splicing of bamboo wire and the frame by drilling the fixed frame, after a close integration of bamboo wire with the fixed frame, and then production of the basic outer frame part of the straight line type bamboo lamp is completed.

\section{B. Curve}

Curve is the product of continuous movement of dots. Compared with the straight line, the curve can give people a sense of movement and rhythm, also has a high degree of freedom, and can produce empty and full changes, giving people a more brisk and active visual experience [6].

Curve type bamboo lamp has a basically same production process and produce with the straight-line type bamboo lamp, and for the main difference in the production process, it needs a bending process of bamboo wire after mechanical drawing or hand-reeling. Due to the high tensile strength and compressive strength of bamboo, bamboo naturally has good toughness, it can bear greater degree of regular bending, without physical and chemical processing, so the shape with lower curvature can be completed directly by hand and connected directly with the structure, to achieve the design and production of curve type bamboo lamps as in "Fig. 7 and "Fig. 8" In production of some bamboo wires with great curvature or irregular bending, the thermal softening or chemical softening method can be used to achieve greater bending of the bamboo wire, expressing the flexible and lively design of works as in "Fig. 9". 


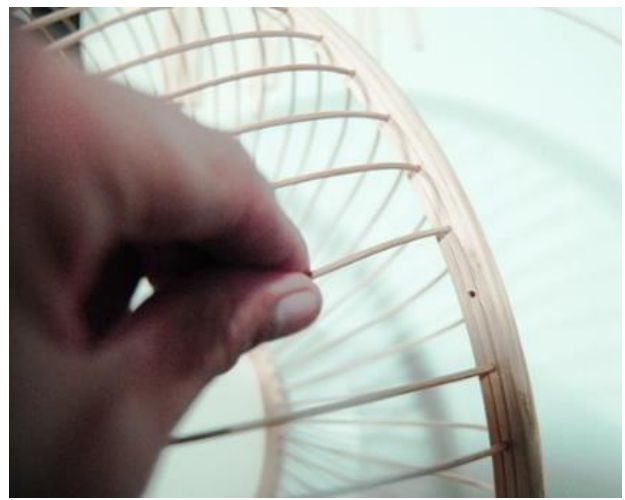

Fig. 7. The combination technology of linear bamboo lamp.

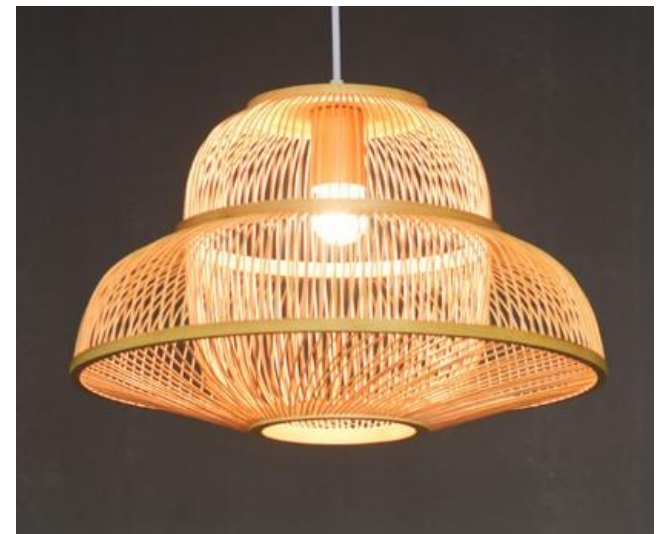

Fig. 8. The curve-type bamboo wire lamps.

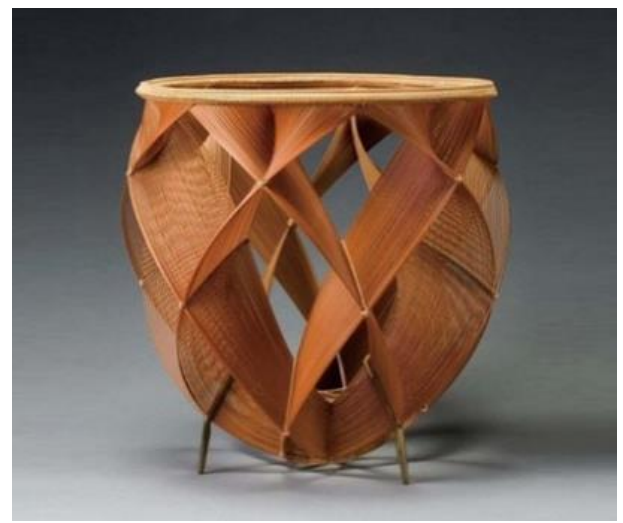

Fig. 9. The bamboo wire table with large curvature.

Different line elements have their unique emotional expression, giving a different psychological experience. The line elements of bamboo include many varieties, such as bamboo wire, bamboo slice, and raw bamboo, which can form line element by different processing. Combined with the principle of formal beauty, the bamboo lamps designed with different line elements have various ways to achieve the technology of production [7].

\section{The Production Process of Facet ElEMENT}

Facet is the track of line movement. Facet has two spaces of length and width, and the various forms of it in the modeling are an important factor of the design. In the design structure, the intersection of lines and the dense formation of dots and lines, form a virtual face; the expansion of dots and lines forms a real face; the segmentation of face, the overlap, splicing and combination of faces form a new face.

\section{A. The Real Surface}

In the biological characteristics of bamboo, there are gaps between the cells and their fiber directions are the same, so when the thickness of the bamboo is relatively thin, it can produce natural translucency. The thin bamboo after slicing can be made into the cover of bamboo lamp, which can maintain the natural texture of bamboo, and in the use of the lamp, the light is more decorative as in "Fig. 10".

Sliced thin bamboo, also known as bamboo skin, is a kind of patch material, which is processed by slicing machine for the bamboo plate. Sliced thin bamboo has a brittle texture, easy to be damaged, and has low strength, so it is often mixed and cohered with non-woven fabric to strengthen the structure, which can make up for its vulnerability and enhance the horizontal tensile strength, and then it can be spliced into a large margin of sliced thin bamboo. Due to the limitation of bamboo skin, the bamboo lamp needs to be glued to the frame during the production of bamboo lamp, and the frame provides the support for the material as shown in "Fig. 11".

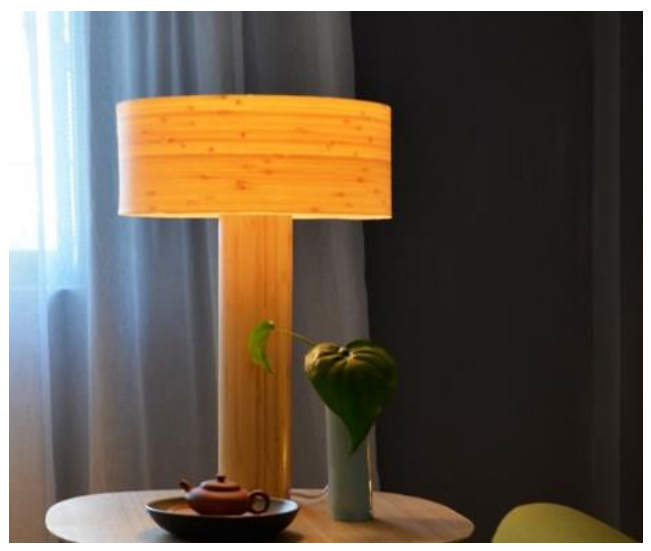

Fig. 10. The table lamp with sliced thin bamboo cover.
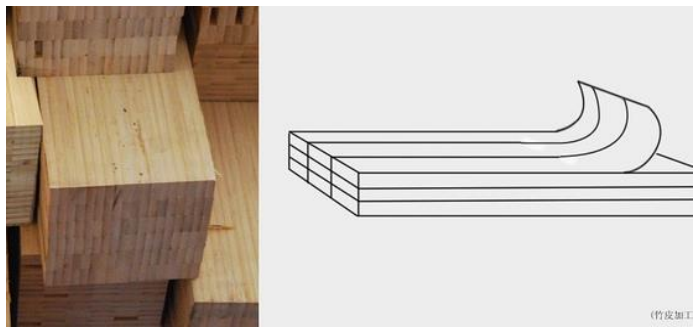

Fig. 11. The sliced thin bamboo craft

\section{B. Virtual Surface}

Virtual surface refers to the face that is made by the intersection of lines and the dense formation of dots and lines, really not existent, but can be perceived. Therefore, in the design of bamboo lamp, the virtual surface often consists of 
densely arranged bamboo wire and bamboo weave as in "Fig. $12 "$ in the production process, it is the same as the bamboo lamps based on line element, and the main difference is the density of bamboo arrangement. In contrast to the former, the bamboo lamp with the virtual surface design has a denser arrangement of bamboo wires. As the bamboo can be woven by crossover, weaving process can be used to complete the production of bamboo lamp cover.

Densely arranged bamboo wires and bamboo weaves can be used as cover of lamp by the manual gap control, which can reduce the discomfort for direct exposure of light source and can form mottled light and shadow, bringing bamboo lamp more lingering charm.

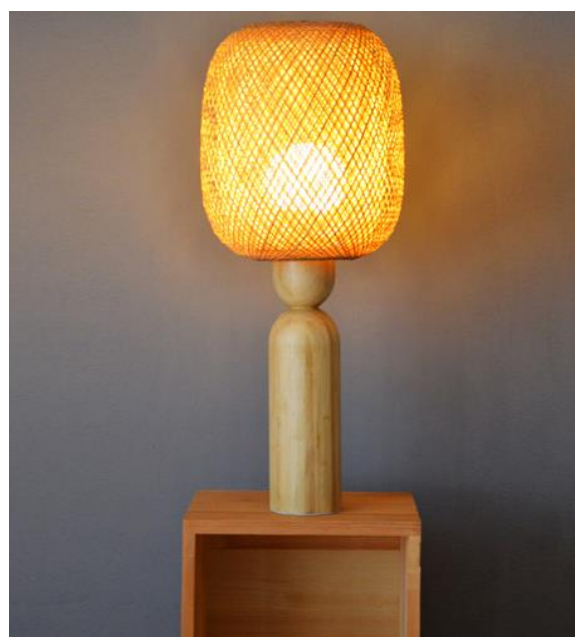

Fig. 12. Multi-layer bamboo table lamp.

\section{The Production Process of Stereo ElEMENT}

Stereo is formed by movement of faces, so the stereo has its data determined and quantified in the three dimensions of length, width and height. In the design of bamboo lamp, the support structure is an objective reflection of stereo element. In the production process, there are two main sources of support structure. One is turning of bamboo plate as in "Fig. 13", the other is the use of raw bamboo "Fig. 14". It is noteworthy that in order to achieve the vertical support structure, natural bending of raw bamboo can be straightened by reverse bending after heating, to achieve the aim; and for the necessary bending support structure, it is also achieved by thermal bending.

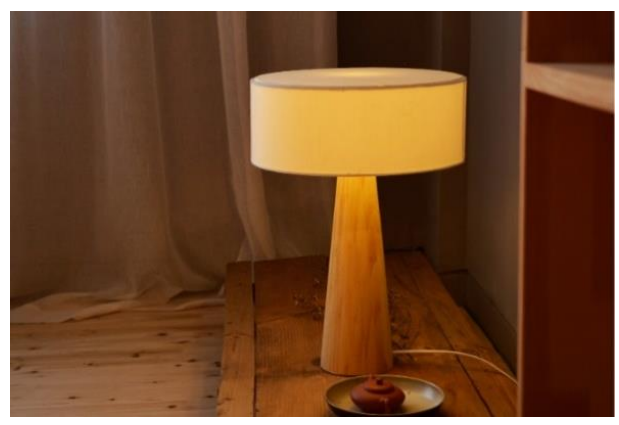

Fig. 13. Bamboo plate lamp.

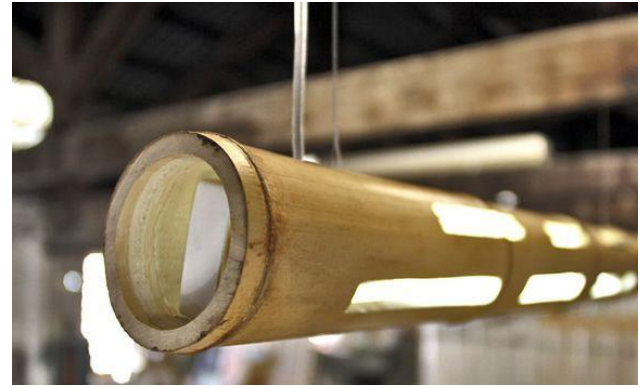

Fig. 14. Raw bamboo lamp.

\section{CONCLUSION}

Product design modeling is based on the form composition, and dot, line, facet and stereo is the basic elements of form composition. By a case analysis and in combination with the theory of composition, this paper made a specific analysis and comparison on the production process of different morphological elements of bamboo lamp, discussed the production process of bamboo lamp based on the morphological elements, and introduced a variety of production processes of bamboo lamps, summed up the production processes of existing bamboo lamp. Bamboo lamp design still has a larger space for development, and the discussion and study on the specific production process of bamboo lamps, provides reference for the innovation of future bamboo lamp design and the new application fields of bamboo.

\section{REFERENCES}

[1] Wang Zhangwang. Basis of Design [M], Mechanical Industry Press, 2009

[2] Gao Jingshu, Zhang Zhongfeng, Zhang Jijuan. Study on Morphological Concept and Elements of Bamboo Lamps and Their Design Performance [J], Packaging Engineering, (03)

[3] Zhang Ying. Study on Design Performance of Bamboo [D], Central South University of Forestry and Technology, 2011

[4] Wu Xiang. Morphology of Design [M], Chongqing University Press, 2008

[5] Zhang Kun. Simple Personality and Fashion -Linear Regression of Product Design [J], Beauty and Times, 2006 (12)

[6] MAO Mengyan. Analysis of the emotional expression of curve modeling in product design [J], Journal of Zhejiang Shuren University, $2016(03)$

[7] Gao Jingshu. Study on Form Design of Bamboo Lamp[D], Master's Degree Thesis from Central South University of Forestry and Technology, 2014. 